\title{
О КУРСЕ «НЕЛИНЕЙНЫЕ УРАВНЕНИЯ В ЧАСТНЫХ ПРОИЗВОДНЫХ» В СИСТЕМЕ ОБУЧЕНИЯ БАКАЛАВРОВ НАПРАВЛЕНИЯ «ПЕДАГОГИЧЕСКОЕ ОБРАЗОВАНИЕ (С ДВУМЯ ПРОФИЛЯМИ ПОДГОТОВКИ МАТЕМАТИКА И ФИЗИКА)»
}

\section{ON THE COURSE "NONLINEAR PARTIAL \\ DIFFERENTIAL EQUATIONS" IN THE \\ BACHELOR'S EDUCATION SYSTEM OF THE DIRECTION “PEDAGOGICAL EDUCATION (WITH TWO TRAINING PROFILES MATHEMATICS AND PHYSICS)" \\ L. Mironova \\ R. Galieva \\ L. Shakirova}

Summary: The article substantiates the possibility of introducing the course «Nonlinear partial differential equations» for bachelors of 44.03 .05 - pedagogical education (with two training profiles: mathematics and physics). The structure and possible content of the corresponding elective course is described. Examples of typical tasks for various sections of the course are given.

Keywords: differential equations, distance learning, elective courses, mathematical models, solitons, educational resource.

Миронова Любовь Борисовна

к.ф.-м.н., дочент, Казанский (Приволжский) федеральный университет, Елабужский институт (г. Елабуга)

Ibmironova@yandex.ru

Галиева Розалина Раяновна

Казанский (Приволжский) фредеральный университет», Елабужский институт, (2. Елабуга)

yarozalina@mail.ru

Шакирова Лейля Рафаковна

Казанский (Приволжский) федеральный университет», Елабужский институт, (г. Елабуга) lylyshakirova@mail.ru

Аннотация: В статье обосновывается возможность введения курса «Нелинейные уравнения в частных производных» для бакалавров направления 44.03 .05 - педагогическое образование (с двумя профилями подготовки: математика и физика). Описывается структура и возможное содержание соответствующего курса по выбору. Приведены примеры типовых задач для различных разделов курса.

Ключевые слова: дифференциальные уравнения, дистанционное обучение, курс по выбору, математические модели, солитоны, образовательный ресурс.

\section{Введение}

$\Pi$ роблемам преподавания дифференциальных уравнений в вузах для различных специальностей в последнее время уделяется пристальное внимание [1-5].

На факультете математики и естественных наук в Елабужском институте Казанского федерального университета студенты, обучающиеся по направлению 44.03 .05 (педагогическое образование с двумя профилями подготовки - математика и физика) изучают стандартный для данной специальности курс «Дифференциальные уравнения», включающий следующие разделы: элементы общей теории обыкновенных дифференциальных уравнений и начала теории линейных обыкновенных дифференциальных уравнений. В современной математике дифференциальные уравнения являются одним из наиболее важных и актуальных разделов математики. В связи с возможностями применения дифференциальных уравнений в математическом моделировании задач из различных областей науки и техники, а также их связью с другими разделами математики, целесообраз-

ным представляется введение курса по выбору, целью которого является ознакомление с современными и актуальными приложениями дифференциальных уравнений. Отметим, что целевой аудиторией курса являются будущие учителя математики и физики, которые должны иметь достаточно широкий кругозор в рассматриваемой области знания. В качестве такого дополнения можно предложить курс, посвященный изучению нелинейных уравнений в частных производных, а также некоторых нелинейных моделей математической физики. Это направление теории дифференциальных уравнений бурно развивалось в XX веке и имеет многочисленные приложения в современной науке и технике (оптоволоконная связь, нервные импульсы, теория волн на воде, в том числе цунами, эффект Джозефсона и др. [6-8]).

Разработанные нами учебно-методические материалы могут послужить основой для создания электронного образовательного ресурса, предназначенного для дистанционного использования учащимися. Дистанционному обучению, в том числе математики в высшей школе, в последнее время уделяется много внимания, этому направлению посвящены, например, работы[9-14]. 
Отметим, что структура курса такова, что от слушателя не требуется ничего, кроме знания основ математического анализа и теории обыкновенных дифференциальных уравнений. В частности, не требуется знакомство с теорией линейных дифференциальных уравнений второго порядка и методами гармонического анализа.

\section{Описание курса}

Разработанный курс состоит из двух разделов.

I. Уравнения с частными производными первого порядка.

II. Нелинейные уравнения математической физики.

Первый раздел включает в себя следующие составные части.

1. Линейные однородные уравнения в частных производных первого порядка.

2. Квазилинейные уравнения первого порядка.

3. Системы двух совместных уравнений первого порядка. Условие совместности системы, интегрирование системы двух уравнений.

4. Уравнение Пфаффа, условия его интегрируемости.

5. Полный, общий и особый интегралы уравнения с частными производными первого порядка. Вводятся понятия полного, общего, особого интеграла и характеристик.

6. Метод Лагранжа-Шарпи нахождения полного интеграла. Излагается метод Лагранжа-Шарпи нахождения полного интеграла, который заключается в нахождении второго уравнения системы при условии, что система из двух уравнений вполне интегрируема.

7. Метод Коши для двух независимых переменных. Здесь рассматриваются: определение характеристических линий на интегральной поверхности, дифференциальные уравнения характеристик первого порядка, определение характеристик из дифференциальных уравнений, построение интегральной поверхности из характеристик, задача Коши.

8. Метод Коши для $\mathrm{n}$ независимых переменных.

Приведем некоторые типичные задачи для раздела I.

1. Проверить условия интегрируемости и найти интегральное многообразие уравнения

$$
\left(y z-z^{2}\right) d x-x z d y+x y d z=0
$$

2. Определить проекции на плоскость $х 0 y$ семейства кривых, определяемых на эллипсоиде $\frac{x^{2}}{a^{2}}+\frac{y^{2}}{b^{2}}+\frac{z^{2}}{c^{2}}=1$ уравнением Пфаффа

$$
x d x+y d y+c\left(1-\frac{x^{2}}{a^{2}}-\frac{y^{2}}{b^{2}}\right)^{\frac{1}{2}} d z=0 .
$$

3. Проинтегрировать

$$
p^{2}+q^{2}+p q-q x-p y-2 z+x y=0 .
$$

4. Для уравнения $z=p x+q y+p q$ найти решение, проходящее через кривую

$$
x=0, z=y^{2} \text {. }
$$

5. Решить задачу Коши для уравнения

$$
z=p q \text {. }
$$

Начальные условия: $x_{0}=u, y_{0}=u^{2}, z_{0}=u^{3}$.

6. Решить задачу Коши для уравнения

$$
p^{2}+q^{2}=1
$$

Начальные условия: $x_{0}=\cos u, y_{0}=\sin u, z_{0}=\frac{1}{2} u$.

\section{Содержание второго разцела}

1. Задача Стефана о фазовом переходе (задача о промерзании).

2. Распространение тепловых возмущений в нелинейных средах.

3. Задача нелинейной теплопроводности с объемным поглощением.

4. Уравнение типа «реакция-диффузия».

5. Уравнение Колмогорова-Петровского-Пискунова

6. Уравнение Бюргерса.

7. Уравнение Кортевега-де Фриза.

Актуальность рассмотренных в разделе II математических моделей несомненна. Например, изучается аналитическое решение одномерной нелинейной задачи теории теплопроводности, которой присвоено имя И. Стефана, поставившего и решившего в 1889 г. задачу о фазовом переходе. Фазовый переход может быть связан с кристаллизацией жидкости при ее охлаждении. В этом случае задачу обычно называют задачей о промерзании, имея в виду, что процесс замерзания воды при ее охлаждении относится к процессам такого класса. К такому роду задачам приходят при моделировании процессов зонной плавки, направленной кристаллизации, получения полупроводников с заданными свойствами.

Исследуя математическую модель процесса эволюции биологического вида, А.Н. Колмогоров, И.Г. Петровский и Н.С. Пискунов в работе [15] показали, что задача вытеснения одного биологического вида другим доминантным видом на некоторой территории может быть сведена к решению нелинейного параболического уравнения с нелинейным младшим членом (уравнение Колмогорова-Петровского-Пискунова)

$$
\frac{\partial u}{\partial t}=\frac{\partial^{2} u}{\partial x^{2}}+k u(1-u), t>0, x \in R^{1}
$$

Волновые процессы являются эффективным средством передачи энергии и информации. Они широко используются в науке и технике. Поэтому исследование закономерностей распространения волн различной природы является важной и актуальной задачей. Наиболее известным нелинейным уравнением волновых про- 
цессов является уравнение Кортевега - де Фриза

$$
\frac{\partial u}{\partial t}+u \frac{\partial u}{\partial x}+\beta \frac{\partial^{3} u}{\partial x^{3}}=0
$$

Приведем примеры задач, относящихся к разделу II.

1. Опишите механизм «опрокидывания» волн в условиях нелинейного конвективного переноса.

2. Преобразуйте уравнение Кортевега - де Фриза к виду

$$
\frac{\partial u}{\partial t}+6 u \frac{\partial u}{\partial x}+\frac{\partial^{3} u}{\partial x^{3}}=0
$$

3. Покажите, что модифицированное уравнение Кортевега - де Фриза

$$
\frac{\partial u}{\partial t}+u^{2} \frac{\partial u}{\partial x}+\frac{\partial^{3} u}{\partial x^{3}}=0
$$

имеет солитонное решение

В качестве основных источников, послуживших основой данного курса, можно указать [16-18].

\section{ЛИТЕРАТУРА}

1. Чернова Т.В. Проблемы преподавания дифференциальных уравнений в высших учебных заведениях // Человеческий капитал как фактор инновационного развития общества. Сборник статей Международной научно-практической конференции. - 2018. - C. 8-12.

2. Гнедаш Е.С., Сербина Л.И. Преподавание основ теории дифференциальных уравнений в дистанционной форме // Вопросы педагогики. - $2019 .-$ № 5 - 2. - C.85-87.

3. Балабаева Н.П. Профессионально-ориентированный подход к преподаванию раздела «дифференциальные уравнения» студентам экономических направлений // Самарский научный вестник. - 2014. - № 4 (9). - С. $22-25$.

4. Муста Л.Г., Журов Г.Н. К вопросу о преподавании численных методов решения дифференциальных уравнений бакалаврам и магистрантам горного университета // Современное образование: содержание, технологии, качество - 2018. - Т. 1. - С. 134-136.

5. Zeytun A.S., Cetinkaya B., Erbas A.K. Understanding prospective teachers' mathematical modeling processes in the context of a mathematical modeling course // Eurasia Journal of Mathematics, Science and Technology Education. - 2017. - Vol. 13. - Issue 3. - Pp. 691 - 722.

6. Филиппов А.Т. Многоликий солитон. - М.: Наука, 1990. - 288 с.

7. Захаров В.Е., Кузнецов Е.А. Солитоны и коллапсы: два сценария эволюции нелинейных волновых систем // УФН. - 2012. - Т. 182 - № 6 - С. $569-592$.

8. Дианов Е.М. На пороге пета-эры // УФН. - 2013. - Т. 183 - № 5 -С. 511-518.

9. Анисимова Т.И. Организация самостоятельной работы бакалавров средствами дистанционного обучения // Фундаментальные исследования. - 2013. № $11-4 .-$ - . 747-750.

10. Гильманова Г.Р., Миронов А.Н., Миронова Л.Б. 0 месте курса по выбору «Элементы теории специальных функций» в системе обучения бакалавров направления «Математика и компьютерные науки» // Современные проблемы науки и образования. - 2017. - № 6. http://www.science-education.ru/ru/ article/view?id=27259

11. Анисимова Т.И., Ганеева А.Р. Возможности электронных образовательных ресурсов при организации самостоятельной работы // EuropeanSocialScienceJournal (Европейский журнал социальных наук). - 2018. - Т. 1 - № 5. - C. 116-122.

12. Arnold R. Will distance disappear in distance studies? Preliminary considerations on the didactic relevance of proximity and distance. // Journal of Distance Education. - 1999. - Vol. 2 - Issue 14 - Pp. 1-9.

13. Guri-Rosenblit, S. "Distance education" and "e-learning": Not the same thing.// Higher Education. - 2005. - Vol. 49 - Pp. 467-493.

14. Guri-Rosenblit, S. Eight paradoxes in the implementation process of E-learning in higher education. // Higher Education Policy. - 2005 - Vol. 18 -Issue 1. - Pp. $5-29$.

15. Колмогоров А.Н., Петровский И.Г., Пискунов Н.С. Исследование уравнения диффузии, соединенной с возрастанием вещества, и его применение к одной биологической проблеме // Бюллетень МГУ. Сер. А. Математика и Механика. - 1937 - Т.1 - № 6 -С. 1-26.

16. Мартинсон Л.К., Малов Ю.И. Дифференциальные уравнения математической физики. - М.: изд-во МГТУ им. Н.Э. Баумана. $-2011 .-367$ с.

17. Колоколов И.В., Кузнецов Е.А., Мильштейн А.И., Подивилов Е.В., Черных А.И., Шапиро Д.А., Шапиро Е.Г. Задачи по методам математической физики. М.: Либроком. - 2013. -288 с.

18. Шарма Дж. Н., Сингх К. Уравнения в частных производных для инженеров. - М.: Техносфера. - 2002. - 320 с.

( Миронова Любовь Борисовна (Ibmironova@yandex.ru), Галиева Розалина Раяновна (yarozalina@mail.ru) Шакирова Лейля Рафаковна (lylyshakirova@mail.ru). 